\title{
Idiopathic Pulmonary Haemosiderosis in a Newborn
}

\author{
C. S. LIVINGSTONE and B. BOCZAROW \\ From Staincliffe General Hospital, Dewsbury, Yorkshire
}

Idiopathic pulmonary haemosiderosis is an uncommon disease. Its symptoms in childhood and adult life are well documented, but diagnosis in infancy may be difficult. We present a fatal case in a newborn infant.

\section{Case Report}

A male infant of $3 \mathrm{~kg}$. was born of a normal pregnancy. Following his discharge from hospital a week after birth, his mother found that he was breathless and slow to feed. $\mathrm{He}$ was examined the following week and the only abnormality found was hypotonia. When $3 \frac{1}{2}$ weeks old he developed oedema of the extremities, followed 3 days later by generalized oedema. On admission he was exhausted, generally hypotonic, and dyspnoeic, but not cyanosed. The chest was clear, heart normal apart from a loud single pulmonary second sound, and liver palpable $3 \mathrm{~cm}$. below the costal margin. ECG showed peaked pulmonary $P$ waves in the chest leads, and evidence of increased activity of the right ventricle. Chest radiograph normal. Hb 13.1 g. $/ 100 \mathrm{ml}$., WBC $8000 /$ c.mm., normal differential. Urinalysis, serum proteins, blood urea, and electrolytes, normal. He was treated with digoxin and frusemide, and initially improved. After three days he relapsed, and was given steroids without effect. He died two days later aged 5 weeks.

Necropsy. The body was slightly oedematous. There were no skin haemorrhages. The lungs were heavy and firm, there were haemorrhagic patches on the pleural surfaces, and cut surfaces revealed irregular areas of haemorrhagic consolidation. Sections of these areas showed alveoli containing masses of red cells, and numerous haemosiderin-laden macrophages which gave a positive reaction for iron. The paratracheal lymph nodes were congested. Their sinuses were wide and

Received November 21, 1966. also contained haemosiderin-laden macrophages. The heart, large blood vessels, abdominal viscera, and other organs showed no unusual features.

\section{Discussion}

Idiopathic pulmonary haemosiderosis has been recognized for over a century, as Virchow reported in 1864, 'brown lung induration in young girls' without heart disease (Soergel, 1957). Soergel and Sommers (1962) reviewed 112 case histories: 91 were less than 16 years old, and the youngest was 4 months. Death occurred in about one-third of patients by 3 years and 4 months and in a further third of the patients at varying intervals. Most deaths were a result of acute pulmonary haemorrhage, but after several years some of the patients developed right-sided heart failure.

Our patient developed symptoms at 1 week, and at $3 \frac{1}{2}$ weeks presented as right heart failure. The loud pulmonary second sound on auscultation and the ECG findings suggested pulmonary hypertension. Although he was dyspnoeic, his chest was clinically and radiographically clear. The history was also unusual in as much as there were no preceding chest symptoms or haemoptysis. The cardiac signs suggested idiopathic pulmonary hypertension, and it was only at necropsy that the correct diagnosis was revealed.

\section{Summary}

A fatal case of idiopathic pulmonary haemosiderosis in a newborn is described.

\section{REFERENCES}

Soergel, K. H. (1957). Idiopathic pulmonary hemosiderosis, review and report of 2 cases. Pediatrics, 19, 1101.

-, and Sommers, S. C. (1962). Idiopathic pulmonary haemosiderosis and related syndromes. Amer. F. Med., 32, 499. 